\title{
The Safety of Therapeutic Monoclonal Antibodies: Implications for Cancer Therapy Including Immuno-Checkpoint Inhibitors
}

\author{
R. DEMLOVA ${ }^{1,2}$, D. VALÍK ${ }^{1,2}$, R. OBERMANNOVA ${ }^{1,2}$, L. ZDRAŽILOVÁ-DUBSKÁ ${ }^{1,2}$ \\ ${ }^{1}$ Department of Pharmacology, Faculty of Medicine, Masaryk University Brno, Czech Republic, \\ ${ }^{2}$ Masaryk Memorial Cancer Institute, Brno, Czech Republic
}

Received September 6, 2016

Accepted October 24, 2016

\begin{abstract}
Summary
Monoclonal antibody-based treatment of cancer has been established as one of the most successful therapeutic strategies for both hematologic malignancies and solid tumors. In addition to targeting cancer antigens antibodies can also modulate immunological pathways that are critical to immune surveillance. Antibody therapy directed against several negative immunologic regulators (checkpoints) is demonstrating significant success in the past few years. Immune checkpoint inhibitors, ipilimumab, pembrolizumab and nivolumab, have shown significant clinical benefit in several malignancies and are already approved for advanced melanoma and squamous NSCLC. Based on their mechanism of action, these agents can exert toxicities that are unlike conventional cytotoxic chemotherapy, whose nature is close to autoimmune diseases - immune related adverse events (irAEs). In this review we focus on the spectrum of irAEs associated with immune checkpoint antibodies, discussing the pharmacological treatment strategy and possible clinical impact.
\end{abstract}

\section{Key words}

Cancer treatment • Monoclonal antibodies • Immune checkpoint inhibitors • Immune related adverse events

\section{Corresponding author}

R. Demlova, Department of Pharmacology, Faculty of Medicine, Kamenice 5, 62500 Brno, Czech Republic. Email: demlova@med.muni.cz

\section{Monoclonal antibodies in cancer}

Monoclonal antibodies (mAbs) are a rapidly growing class of human therapeutics representing approximately $25 \%$ of drugs under development (Datamonitor: Pipeline insights 2009). By 2014, 34 therapeutic mAbs are predicted to be on the market for treating cancer, autoimmune diseases, and infectious diseases (Datamonitor: Pipeline insights 2009). Since their discovery in 1975, mAbs have been described as "magic bullets" with the potential to seek out and bind targets with high affinity and specificity (Kohler et al. 1975). Cancer diseases are one of the major groups where monoclonal antibodies are used in clinical practice. There have been twelve antibodies that have received approval from the FDA for the treatment of a variety of solid tumors and hematological malignancies in 2014 (Scott et al. 2012). In addition, there are a large number of additional therapeutic antibodies that are currently being tested in early- and late-stage clinical trials. The most common type of mAbs used to treat cancer are "naked mAbs". Most naked mAbs attach to antigens on cancer cells, but some work by binding to antigens on other, non-cancerous cells, or even free-floating proteins. We can simplify three major mechanism of actions of naked mAbs. One principle is boosts a person's immune response against cancer cells by attaching to them and acting as a marker for the body's immune system to destroy them. An example is alemtuzumab, which binds to the CD52 antigen on lymphocytes and is used to treat some patients with chronic lymphocytic leukemia (CLL). Another naked mAbs work mainly by attaching to and blocking antigens on cancer cells that help cancer cells grow or spread. For example, trastuzumab is an antibody against the HER2 protein. Currently the most researched $\mathrm{mAbs}$ are naked mAbs which boost the immune response 
by targeting immune system checkpoints like anti-CTLA-4 monoclonal antibody ipilimumab or nivolumab and pembrolizumab targeting programmed cell death 1 receptor (PD-1). Adverse effects of this immune checkpoint inhibitors will be discussed in this review in more detail.

In cancer therapy apart from the naked Mabs we can use also conjugated monoclonal antibodies, mAbs joined to a chemotherapy drug or to a radioactive particle. Conjugated mAbs are also sometimes referred to as tagged, labeled, or loaded antibodies. Ibritumomab tiuxetan is an example of a radiolabeled mAb. Antibodydrug conjugates (ADCs) like brentuximab vedotin targets the CD30 antigen, trastuzumab emtansine (also called TDM-1) is an antibody that targets the HER2 protein attached to a chemo drug called DM1. It's used to treat some breast cancer patients whose cancer cells have too much HER2. Bispecific monoclonal antibodies are made up of parts of 2 different mAbs, meaning they can attach to 2 different proteins at the same time. An example is blinatumomab, which is used to treat some types of acute lymphocytic leukemia (ALL). One part of blinatumomab attaches to the CD19 protein, which is found on some leukemia and lymphoma cells. Another part attaches to CD3, a protein found on immune cells called T cells. By binding to both of these proteins, this drug brings the cancer cells and immune cells together, which is thought to cause the immune system to attack the cancer cells.
Compared with chemotherapy drugs, monoclonal antibodies tend to have fewer serious side effects, however, as with all agents, administration of mAbs should be associated with adverse events (AEs) as a result of enhancing/inhibiting the activity of the target molecule on the target tissue, or due to interactions of the $\mathrm{mAb}$ with target molecules on tissues other than the intended ones (Catapano et al. 2013).

\section{Classification of adverse effects of biological agents}

Traditionally, adverse reactions should be subclassified according to their action (Naisbitt et al. 2000) (Table 1): so-called type A reactions correspond to the pharmacological activity of the drug, and are thus predictable (Hoigne et al. 1993). About $16 \%$ of side effects are type B reactions (Naisbitt et al. 2000), which are not related to the pharmacological activity of the drug and are nonpredictable. The majority of type $\mathrm{B}$ reactions are immune-mediated side-effects like hypersensitivity reactions. Types $\mathrm{C}, \mathrm{D}$, and $\mathrm{E}$ are not mechanisms but characteristics of their manifestations; they are not referred to frequently in the literature. The letter $\mathrm{C}$ refers to continuous, chronic. Type $\mathrm{D}$ refers to delayed in appearance, making them difficult to diagnose. Type $\mathrm{E}$ refers to end of use, F means failure of therapy (Edwards et al. 2000).

Table 1. Classification of adverse drug reactions (Naisbitt et al. 2000).

\section{Type A (augmented) reactions}

Type B (bizarre) reactions

Type C (chronic) reactions

Type D (delayed) reactions

Type E (end of treatment) reactions
Predicted from the known pharmacology of the drug. These reactions are dose-dependent: examples are bleeding with anticoagulants

Reactions are not predicted from the known pharmacology of the drug. They appear (but actually are not) relatively dose-independent, as very small doses might already elicit symptoms. They include immune-mediated side-effects like maculopapular exanthema, but also other hypersensitivity reactions, like aspirin-induced asthma

Which are related to the chemical structure and its metabolism, e.g. paracetamol hepatotoxicity

Which appear after many years of treatment, e.g. bladder carcinoma after treatment with cyclophosphamide

Occur after drug withdrawal, e.g. seizures after stopping phenytoin
Biological agents differ from most drugs as they are not small chemical compounds (xenobiotics) but are proteins produced in a way to make them as similar to human proteins as possible. They are not metabolized like drugs but are processed like other proteins, with the differences in pharmacokinetic as well as pharmacodynamic properties. Thus, adverse reactions to biological agents might differ from those elicited by 
classical drugs. More appropriate classification of mAbs adverse effects is a subclassification published by Pichler et al. (2006) based on mechanism of action and structure, as illustrated in Figure 1. To distinguish it from the classification of side-effects to chemicals/drugs (Table 1), the Greek alphabet is used for the five types (type $\alpha, \beta, \gamma$, $\delta$ and $\varepsilon$ ). Type $\alpha$ (high cytokine and cytokine release syndrome) are side-effects connected to the systematic application of cytokines in relatively high doses or to high concentrations of cytokines released into the circulation (Vasquez et al. 1995). Type $\beta$ reactions can be termed as hypersensitivity. Thereby basically three forms of allergies can be differentiated: IgE-, IgG- and T cellmediated reactions. Type $\gamma$ immune (cytokine) imbalance syndromes have immunological features, but cannot be explained by high cytosine levels or typical hypersensitivity reactions. As illustrated in Figure 1, these reactions can be further subdivided in, impaired functions, and unmasking or causing an immune imbalance leading to autoimmune, auto-inflammatory or allergic reactions. Type $\delta$ (cross-reactivity) might be that antibodies generated to an antigen expressed on tumour cells might also cross-react with normal cells, which express this structure as well, albeit to a lower degree (Perez-Soler et al. 2005). Type $\varepsilon$ (non-immunological side-effects) may elicit symptoms not directly related to the immune system, sometimes revealing unknown functions of the biological agents given or targeted.

\begin{tabular}{|c|}
\hline Type $\boldsymbol{\alpha}$ \\
\hline High cytokine \\
\& cytokine \\
release \\
syndrome \\
(anti-CD3) \\
\hline
\end{tabular}

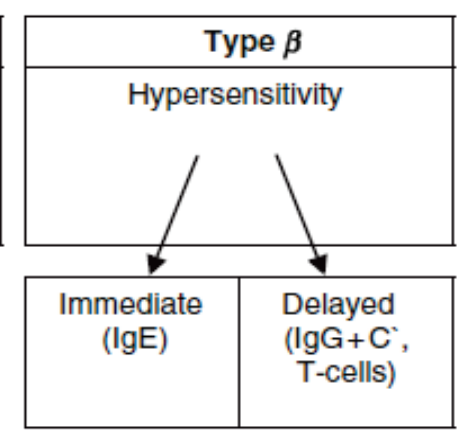

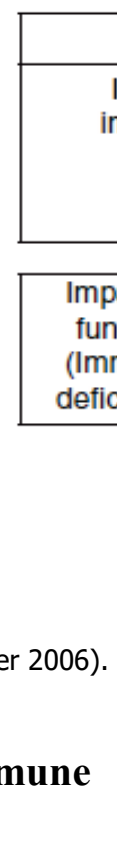
related adverse events (irAEs)

Cancer therapy based on monoclonal antibodies against checkpoints of immune reaction is today considered a breakthrough method in oncology. Results with the anti-CTLA-4 (cytotoxic $\mathrm{T}$ lymphocytic antigen-4) antibody ipilimumab in the treatment of advanced malignant melanoma have represented a revolution in anti-tumour therapy and been a catalyst for developing new antibodies focusing on other control molecules (checkpoints) (Lakomy et al. 2015) What is especially worth mentioning in this context are the antibodies against programmed cell death receptor (PD-1) - nivolumab and pembrolizumab and against its ligand (PD-L1), BMS-936559, MPDL3280A or lambrolizumab ((Weber et al. 2015, Hamid et al. 2013)). The development and use of immune therapy have achieved their greatest progress so far in advanced melanoma, but soon we will be similarly treating non-small-cell pulmonary carcinoma and other tumour types.

These antibodies prevent an inhibition of interaction between PD-1 and PD-L1/L2, on the other hand cause secondary potentiation of the effector component of immunity on the "peripheral" level directly in the tumour tissue. Break of tolerance towards the tumour may, however, be accompanied by unwanted break of tolerance towards "normal tissues", which leads to adverse reactions whose nature is close to autoimmune diseases - immune related adverse events (irAEs). IrAEs include dermatologic, GI, hepatic, endocrine, and other less common inflammatory events. Because irAEs likely arise from general immunologic enhancement, temporary immunosuppression with corticosteroids, tumor necrosis factor $\alpha$ antagonists or other agents is often necessary and should follow established algorithms (Postow et al. 2015). Due to the high frequency of irAEs with the risk of life-threatening complications, sufficient education of 
patients, their family members and medical specialists is necessary. Warnings and recommendations have been prepared about how to proceed suspected irAEs. Early commencement of immunosuppressive therapy with corticosteroids is the key step towards management of the incident, reduction of morbidity and potentially also mortality. If corticoids are not sufficiently effective, other immunosuppressants are added, such as infliximab or mycophenolate mofetil (Lakomy et al. 2015).

\section{Side effects of ipilimumab}

Ani-CTLA-4 monoclonal antibody ipilimumab was the first immune checkpoint receptor used in daily clinical practice. The frequency of adverse effects of ipilimumab is relatively high; in a pre-authorization study of the dose of $3 \mathrm{mg} / \mathrm{kg}$ it ranged between $80-90 \%$. Luckily the toxicity was mild to moderate in the vast majority of the cases (grade 1 and 2 toxicity). Serious (grade 3 ) and life-threatening (grade 4) toxicity pursuant to NCI- CTCAE v3.0 (National Cancer Institute Common Terminology Criteria for Adverse Events version 3.0) was reported for about $20-25 \%$ of the study population (Hodi et al. 2010). Adverse effects may already occur in the course of or after the infusion (nausea, vomiting, febrility, pain and vertigo, rash and pruritus), but these reactions are singular and mostly non-serious. The general recommendation is to suspend the infusion until symptoms retreat, with potential administration of antihistaminics or antipyretics, and subsequently to restart at a slower (about half) speed with patient monitoring (Fecher et al. 2013). Premedication with antihistaminics or antipyretics before the following administrations is recommended for consideration in those cases. In the case of serious adverse reaction of levels 3 and 4 (bronchospasm, hypotension, anaphylaxis) the procedure is identical to other hypersensitivity reactions, with the permanent discontinuation of therapy recommended. The irAEs are a bigger concern, though, as they are most common and grow with the dose. In the case of the dose of $3 \mathrm{mg} / \mathrm{kg}$ they appear in up to $60 \%$ of patients, while severe irAE toxicity of levels 3 and 4 has been reported for $10-15 \%$ of the study subjects (Hodi et al. 2010, Wolchok et al. 2010). The most frequent irAEs include dermal toxicity (rash, pruritus), enterocolitis and diarrhea, endocrinopathy (hypophysitis, thyroiditis) and liver test elevation. The immune system may, however, attack any part of the anatomy (heart, lungs, kidneys, nervous system, eyes, hematopoietic system etc.). Biopsy then most often reveals tissue infiltration with T lymphocytes or with neutrophils (Fecher et al. 2013). Over time, we can first expect dermal toxicity (after three weeks), followed by colitis and diarrhea (after five weeks), hepatic toxicity (after $6-7$ weeks) and finally endocrinopathy (after 7-8 weeks from therapy commencement) (Weber et al. 2012). We should also remember that IrAEs may, however, also appear a couple of weeks or months after therapy completion. The text below will deal with the most frequent irAEs and how to address them.

\section{Immune-conditioned dermal toxicity}

Dermal toxicity is one of the most common irAEs $(40-45 \%)$ usually first occurring after week 3 of therapy and culminating in week 6 (Lakomy et al. 2015, Hodi et al. 2010, Weber et al. 2012). This is mostly level 1 toxicity (affecting $<10 \%$ of the body surface) or toxicity level 2 (with an effect on between 10 and $30 \%$ of the body surface). Severe toxicity (levels 3 and 4 ) is luckily rare, only ranging between $1-2 \%$ in the preauthorization study. Usual manifestations include maculopapular exanthema (20\%) and/or pruritus (25\%). Another manifestation may be vitiligo, which is rather cosmetic issue, but the patient must be educated about protection against UV radiation. Blisters are already a manifestation of a severe reaction. Stevens-Johnson syndrome and toxic epidermal necrolysis occur in $<1 \%$ patients and are a reason for permanent discontinuation of therapy.

\section{Immune-conditioned colitis and diarrhea}

Diarrhea induced by ipilimumab is another of the most common autoimmune-conditioned adverse reactions. This effect appears after week 5 of therapy. In the case of an ipilimumab dose of $3 \mathrm{mg} / \mathrm{kg}$, this $\mathrm{AE}$ occurs in about $30 \%$ patients, and incidence rises with the dose $-44 \%$ at $10 \mathrm{mg} / \mathrm{kg}$ i.v. (Hodi et al. 2010, Weber et al. 2012). Severe diarrhea or colitis levels 3 or 4 occur in the case of a $3 \mathrm{mg} / \mathrm{kg}$ dose in about $5-8 \%$ of patients, colitis most often affecting the descending colon (Wolchok et al. 2010). A big threat is potential intestinal perforation, with potential fatal consequences.

Prophylactic administration of oral corticoids (budesonide) did not change the incidence of diarrhea (Weber et al. 2009). The so far published results suggest that immunosuppressive corticoid therapy administered in 
the course of the immunotherapy does not substantially affect the therapeutic effect of ipilimumab (Weber et al. 2009, Harmankaya et al. 2011).

\section{Immune-conditioned hepatic toxicity}

Hepatic toxicity caused by ipilimumab, even though rarer in comparison to the previously mentioned immune-conditioned adverse effects, may also represent a life-threatening reaction. The onset of this irAE usually occurs after week 6 of ipilimumab therapy, becoming very rare after week 14 (Weber et al. 2012). If all recommendations for diagnosis and treatment are observed, the prognosis for patients with this type of irAE is excellent; the liver test results usually normalize very quickly after commencement of the appropriate therapy - in about two weeks (Della Scarpati et al. 2014). A patient with hepatic toxicity may be asymptomatic (laboratory results usually show elevated ALT and/or AST and/or bilirubin), but frequent complaints include febrility, fatigue, nausea, jaundice, changed stool color or urine colour, and sometimes pruritus. Patient examination must include taking a careful medical history to exclude other causes - fungi, alcohol, contact with chemicals or the effect of concurrent medication (such as paracetamol and others). Clinical examination may reveal, in addition to icterus, also hepatomegaly, the patient may be exhausted, or the find may be completely negative. An infectious cause must always be excluded in level 2 and higher of hepatic toxicity, and antibodies against nuclear antigen and mitochondrial antigen must be sampled (ANA, SMA). Exclusion of progression of the malignant disease by imaging methods is also necessary. In the case of level 3 and 4 toxicity, liver biopsy is to be considered (finds typical of hepatic toxicity include infiltration of the liver parenchyma with $\mathrm{T}$ lymphocytes, sometimes with necrosis of hepatocytes) (Lakomy et al. 2015).

\section{Immune-conditioned endocrinopathy}

Like the immune-conditioned hepatopathy endocrinopathy is also less frequent than dermal or gastrointestinal toxicity, but again represents a potentially life-threatening condition. The deceptiveness of endocrinopathy lies in its late onset $(7-8$ weeks after ipilimumab therapy commencement, with the highest incidence between weeks 12 and 24), and in the fact that, unlike hepatic toxicity, the probability of occurrence does not decrease in time from completion of therapy; instead, the appearance of the curve is rather like a plateau (Weber et al. 2012).

Possible endocrinopathies include hypopituitarism (with or without hypophysitis), hypofunction of the adrenals, hypo- or hyperfunction of the thyroid gland and hypofunction of the gonads. In the case of a suspected adverse effect of this kind, the medical history of the patient is again important (incidence of endocrinopathies in the patient's personal and family history), the patient may complain of non-specific symptoms such as fatigue, weakness, febrilia, abdominal pain, vomiting, diarrhea, headaches or sensory disorders. Clinical warning symptoms include signs of dehydration, hypotension or other signs of commencing systemic inflammatory response syndrome. Laboratory examinations, apart from basic internal environment parameters (often hyperkalemia, hyponatremia and hypoglycemia) and blood count, include endocrinopathy screening: sampling of free $\mathrm{T} 3$ and $\mathrm{T} 4, \mathrm{TSH}$, antibodies against thyroidal peroxidase - anti TPO, morning serum cortisol, levels of corticotropin (ACTH), and in males testosterone levels, and in females FSH and LH levels. For differential diagnosis, an ACTH stimulation test may be considered. Indicated imaging methods include MRI of the brain, with a focus on hypophysis and also on the exclusion of brain metastases. It must be mentioned at this point, that there does not exist any general consensus about the therapy and monitoring, and the literature shows conflicting data on the therapy of this adverse effect and the possibility of returning to ipilimumab therapy (Weber et al. 2012, Della Scarpati et al. 2014, O'Day et al. 2010). Repeated consultations and cooperation with an endocrinologist are a priority. If corticoids need to be administered (toxicity levels 3 and 4), then corticoids with mineralocorticoid activity are preferred with slow discontinuation (Lakomy et al. 2015).

\section{Less frequent immune-conditioned adverse effects}

Other possible, although rare (less than $1 \%$ of cases), immune-conditioned adverse effects of ipilimumab include: meningitis, uveitis, pneumonitis, pancreatitis, pericarditis, myocarditis, nephritis, various angiopathies, hemolytic anemias, thrombocytopenia etc. Straightforward therapeutic procedures are not known, where such adverse effects occur therapy is managed like it is for other autoimmune diseases with corticoids as the 
therapy of choice. Generally, level 3 and 4 toxicity is a reason for discontinuing ipilimumab therapy. Details are in the ipilimumab SmPC (EMA 2012).

\section{Immune-conditioned adverse effects of anti- PD-1/PD-L1 antibodies and their combi- nation with ipilimumab}

Toxicity of these antibodies is generally lower than of ipilimumab Topalian et al. 2012, Wolchok et al. 2015). Severe dermal toxicity levels 3 and 4 are exceptional $(2 \%)$. Also, level 3 or 4 diarrhea is uncommon $(1-2 \%)$, as is hepatic toxicity (below $3 \%$ ) and severe endocrinopathy $(<1 \%)$. Unlike ipilimumab, pneumonitis occurs more often after anti-PD-1 antibodies $(<5 \%)$. Therapy of severe pneumonitis is again based on corticoids in high doses (methylprednisolone $2 \mathrm{mg} / \mathrm{kg}$ i.v. $1-2$ times daily) or infliximab. Other toxicities are sporadic, but cannot be neglected either. The principles of therapy of irAEs caused by anti-PD-1/PD-L1 antibodies are the same as with ipilimumab. A considerably higher incidence of irAEs has been described in reports from studies of combinations of anti-PD- 1 and anti CTLA-4 antibodies (nivolumab + ipilimumab). Here the occurrence of severe irAEs of levels 3 and 4 ranged around $50 \%$, with significant representations of gastrointestinal toxicity (15\%) and hepatic toxicity (19\%). A number of these severe toxicities, however, only met the laboratory criteria NCI-CTCAE (such as the elevation of amylase and lipase without clinical signs of pancreatitis). The combination resulted in no new toxicity (Wolchok et al. 2015).

\section{Conclusions}

The development of immune checkpoint inhibitors targeting cytotoxic T-lymphocyte antigen 4 (CTLA-4) and programmed cell death-1 (PD-1) has significantly improved the treatment of a variety of cancers. Although these agents can lead to remarkable responses, their use can also be associated with unique adverse effects. The spectrum of AEs associated with immune checkpoint antibodies are termed immunerelated AEs (irAE's). The underlying pathophysiology relates to the immune-based mode of action of these agents, leading to T-cell inflammatory infiltration of solid organs, and increased serum inflammatory cytokines. IrAEs are frequent side effects of checkpoint inhibitors, they are possibly life-threatening and there is no known predictive biomarker for their occurrence. The most frequent irAEs include dermal toxicity (exanthema, pruritus), GIT toxicity (diarrhea, colitis), endocrine toxicity (hypopituitarism, hypophysitis, hypothyreosis, adrenal insufficiency), liver toxicity (elevation of transaminase, hepatitis), and in connection with anti-PD-1 antibodies, pneumonitis. IrAEs have their onset and duration (kinetics) well described, but since they can even occur several months after therapy completion (endocrinopathy), the safe interval after therapy completion is not known. Incidence of irAEs is higher for ipilimumab (dose-dependent) in comparison to anti-PD-1/PD-L1 antibodies, and even higher for the combination of ipilimumab and nivolumab. Retreat of toxicity may be slower in the case of anti-PD-1/PD-L1 antibodies; therefore long-term follow up is recommended (Weber et al. 2015). The important is the early medication of immunosuppressant with corticoids and their slow discontinuation, if the corticoids show insufficient or no effect, other immunosuppressants must be prescribed. These recommendations for therapy of irAEs are universal for all checkpoint inhibitors, although they are mainly based on experience from clinical studies with ipilimumab. New adverse effects may occur with the introduction of new antibodies and their combinations. The success of therapy with checkpoint inhibitors is conditional not only upon the erudition of the clinician, but also upon having an educated and cooperating patient (Lakomy et al. 2015).

\section{Conflict of Interest}

There is no conflict of interest.

\section{Acknowledgements}

This work was supported by Project of Research Infrastructure LM2015090 (CZECRIN).

\section{References}

CATAPANO AL, PAPADOPOULOS N: The safety of therapeutic monoclonal antibodies: Implications for cardiovascular disease and targeting the PCSK9 pathway. Atherosclerosis 228: 18-28, 2013. 
Datamonitor: Pipeline insight: Biologic targeted cancer therapies - Next generation jostles for market position, DMHC2573, 2009.

DELlA SCARPATI GV, FUSCIELLO C, PERRI F, SABBATINO F, FERRONE S, CARLOMAGNO C, PEPE S: Ipilimumab in the treatment of metastatic melanoma: management of adverse events. Onco Targets Ther 7: 203-209, 2014.

EDWARDS IR, ARONSON JK: Adverse drug reactions: definitions, diagnosis, and management. Lancet 356: 1255-1259, 2000.

EMA (European Medicines Agency) 2012, http://www.ema.europa.eu/docs/cs_CZ/document_library/EPAR__Product_Information/human/002213/WC500109299.pdf.

FECHER LA, AGARWALA SS, HODI FS, WEBER JS: Ipilimumab and its toxicities: a multidisciplinary approach. Oncologist 18: 733-743, 2013.

HAMID O, ROBERT C, DAUD A, HODI FS, HWU WJ, KEFFORD R, WOLCHOK JD, HERSEY P, JOSEPH RW, WEBER JS, ET AL.: Safety and tumor responses with lambrolizumab (anti-PD- 1) in melanoma. $N$ Engl J Med 369: 134-144, 2013.

HARMANKAYA K, ERASIM C, KOELBLINGER C, IBRAHIM R, HOOS A, PEHAMBERGER H, BINDER M, ET AL.: Continuous systemic corticosteroids do not affect the ongoing regression of metastatic melanoma for more than two years following ipilimumab therapy. Med Oncol 28: 1140-1144, 2011.

HODI FS, O'DAY SJ, MCDERMOTT DF, WEBER RW, SOSMAN JA, HAANEN JB, GONZALEZ R, ROBERT C, SCHADENDORF D, HASSEL JC, ET AL.: Improved survival with ipilimumab in patients with metastatic melanoma. N Engl J Med 363: 711-723, 2010.

HOIGNE R, SCHLUMBERGER HP, VERVLOET D, ZOPPI M: Epidemiology of allergic drug reactions. Monogr Allergy 31: 147-170, 1993.

KOHLER G, MILSTEIN C: Continuous cultures of fused cells secreting antibody of predefined specificity. Nature 256: 495-497, 1975.

LAKOMY R, POPRACH A: Side-effects of modern immunotherapy and how to solve them in the clinics. Klin Onkol 28 (Suppl 4): 4S103-4S114, 2015.

NAISBITT DJ, GORDON SF, PIRMOHAMED M, PARK BK: Immunological principles of adverse drug reactions: the initiation and propagation of immune responses elicited by drug treatment. Drug Saf 23: 483-507, 2000.

O'DAY SJ, MAIO M, CHIARION-SILENI V, GAJEWSKI TF, PEHAMBERGER H, BONDARENKO IN, QUEIROLO P, LUNDGREN L, MIKHAILOV S, ROMAN L, ET AL.: Efficacy and safety of ipilimumab monotherapy in patients with pretreated advanced melanoma: a multicenter single-arm phase II study. Ann Oncol 21: 1712-1717, 2010.

PEREZ-SOLER R, SALTZ L: Cutaneous adverse effects with HER1/EGFR-targeted agents: is there a silver lining? J Clin Oncol 28: 5235-5246, 2005.

PICHLER WJ: Adverse side-effects to biological agents. Allergy 61: 912-920, 2006.

POSTOW MA, CALLAHAN MK, WOLCHOK JD: Immune checkpoint blockade in cancer therapy. J Clin Oncol 33: 1974-1982.

SCOTT AM, ALLISON JP, WOLCHOK JD: Monoclonal antibodies in cancer therapy. Cancer Immun 12: $14,2012$.

TOPALIAN SL, HODI FS, BRAHMER JR, GETTINGER SN, SMITH DC, MCDERMOTT DF, POWDERLY JD, CARVAJAL RD, SOSMAN JA, ATKINS MB, ET AL.: Safety, activity, and immune correlates of anti-PD-1 antibody in cancer. N Engl J Med 366: 2443-2454, 2012.

VASQUEZ EM, FABREGA AJ, POLLAK R: OKT3-induced cytokine-release syndrome: occurrence beyond the second dose and association with rejection severity. Transplant Proc 27: 873-874, 1995.

WEBER JS, D'ANGELO SP, MINOR D, HODI FS, GUTZMER R, NEYNS B, HOELLER C, KHUSHALANI NI, MILLER WH JR, LAO CD, ET AL.: Nivolumab versus chemotherapy in patients with advanced melanoma who progressed after anti-CTLA- 4 treatment (CheckMate 037): a randomised, controlled, open-label, phase 3 trial. Lancet Oncol 16: 375-384, 2015.

WEBER JS, KAHLER KC, HAUSCHILD A: Management of immune- related adverse events and kinetics of response with ipilimumab. J Clin Oncol 30: 2691-2697, 2012. 
WEBER J, THOMPSON JA, HAMID O, MINOR D, AMIN A, RON I, RIDOLFI R, ASSI H, MARAVEYAS A, BERMAN D, ET AL.: A randomized, double-blind, placebo-controlled, phase II study comparing the tolerability and efficacy of ipilimumab administered with or without prophylactic budesonide in patients with unresectable stage III or IV melanoma. Clin Cancer Res 15: 5591-5598, 2009.

WEBER JS, YANG JC, ATKINS MB, DISIS ML: Toxicities of immunotherapy for the practitioner. J Clin Oncol 33: 2092-2099, 2015.

WOLCHOK JD, CHIARION-SILENI V, GONZALEZ R, RUTKOWSKI P, GROB JJ, COWEY CL, LAO CD, SCHADENDORF D, FERRUCCI PF, SMYLIE M, ET AL.: Efficacy and safety results from a phase III trial of nivolumab (NIVO) alone or combined with ipilimumab (IPI) versus IPI alone in treatment- naive patients (pts) with advanced melanoma (MEL) (CheckMate 067). J Clin Oncol 33 (Suppl: abstr. LBA1), 2015.

WOLCHOK JD, NEYNS B, LINETTE G, NEGRIER S, LUTZKY J, THOMAS L, WATERFIELD W, SCHADENDORF D, SMYLIE M, GUTHRIE T, ET AL.: Ipilimumab monotherapy in patients with pretreated advanced melanoma: a randomized, double-blind, multicentre, phase 2, dose-ranging study. Lancet Oncol 11: $155-164,2010$. 